\title{
Mesenchymal Stem Cells and Periodontal Regeneration
}

\author{
Francis J. Hughes
}

Published online: 3 January 2014

(C) Springer International Publishing AG 2014

\begin{abstract}
The use of mesenchymal stem cell (MSC) therapy offers the potential to develop a completely novel and improved method of periodontal regeneration compared to existing methods. Since the initial demonstration of the presence of MSCs in the periodontal ligament, many recent studies have now demonstrated the potential for the transplantation of MSCs from PDL and other sources to enhance periodontal regenerative outcomes in a variety of animal models. In addition, the recent demonstration of the possible utility of allogeneic MSCs and MSCs derived from inducible pluripotent stem cells may offer new methods of delivering such therapies. Determination of the specific roles that MSCs may play in enhancing regenerative outcomes requires further investigation. The principle of MSC-directed periodontal regenerative therapy is accepted in the field, but extensive investigation is necessary to establish viable, efficacious, and practically applicable human therapies.
\end{abstract}

Keywords Mesenchymal stem cell $\cdot$ Periodontal ·

Regeneration · Tissue engineering $\cdot$ Bone marrow $\cdot$ Dental stem cells $\cdot$ Inducible pluripotent stem cells, Allogeneic ·

Periodontal ligament $\cdot$ Cementum $\cdot$ Bone .

Immunomodulation $\cdot$ Scaffolds

\section{Introduction}

Periodontal regeneration, the restoration of the periodontal tissues destroyed by periodontitis, has been an important objective of periodontal therapies for many years. Existing regenerative therapies have considerable limitations, most

F. J. Hughes $(\bowtie)$

Dental Institute, Kings College London, Floor 21 Tower Wing, Guys

Hospital, London SE1 9RT, UK

e-mail: francis.hughes@kcl.ac.uk notably that they are applicable in only very specific clinical situations and are generally considered to provide limited, unpredictable outcomes. Consequently, there continues to be considerable research activity in an attempt to improve these therapies and to develop novel treatments to address this issue.

With the advent of tissue engineering, there is great interest in the potential therapeutic application of stem cells for periodontal regeneration, and there has been a marked increase in publications in this area over the past 5 years. However, there remain many basic scientific, translational, clinical, and practical issues that must be addressed before this research activity can be translated into viable therapies. Some of these research questions are summarized in Table 1. This article reviews recent research into the potential application of mesenchymal stem cells (MSCs) for periodontal regeneration, with particular emphasis on how these studies may advance the field by addressing some of the questions set forth in Table 1. We focus particularly (but not exclusively) on the potential use of periodontal ligament-derived MSCs, as these have attracted the most attention in the field. In many respects, this is a relatively recent and novel area of research, and as such, it is inevitable that much of the recent research addresses some of the more fundamental biological questions listed in Table 1.

\section{Current Status of Periodontal Regeneration - The Context for Developing Stem Cell Therapies}

Although the normal outcome of healing following treatment of periodontitis is tissue repair, with little or no restoration of the previously damaged tissues, it has long been suggested that the periodontal ligament has high inherent regenerative potential [1-3] and can give rise to osteoblasts, periodontal ligament (PDL) fibroblasts, and cementoblasts. More recently, this concept is supported by the demonstration of the presence of stem cells within the periodontal ligament $[4,5]$. 
Table 1 Some of the basic scientific, translational, clinical, and practical questions to be addressed in studies oninto the application of mesenchymal stem cells for periodontal regeneration.

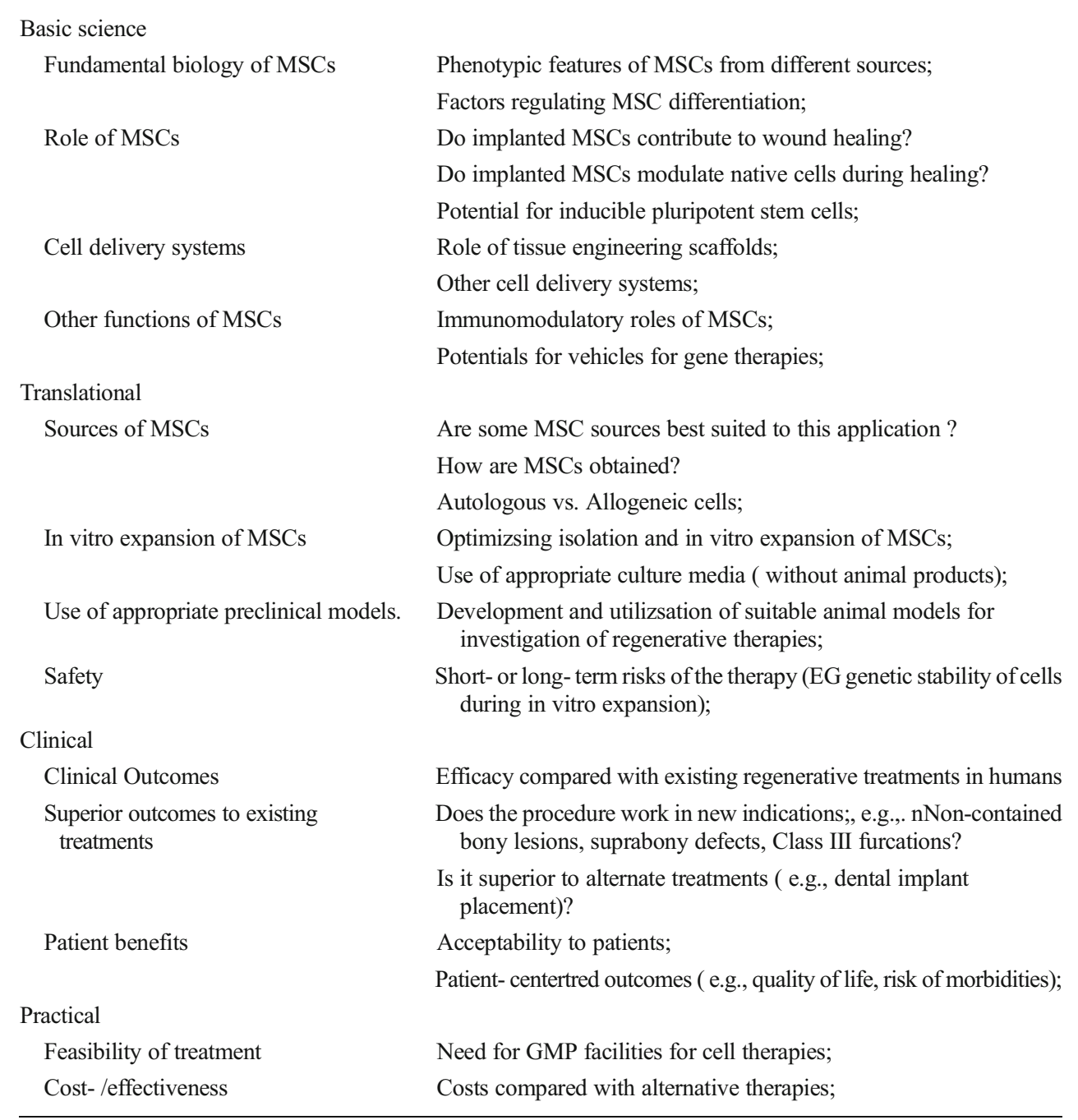

Biologically, current regenerative therapies such as guided tissue regeneration (GTR), grafting, and application of biological agents can be considered as attempting to 'unlock' this inherent regenerative potential. In the case of GTR, the placement of a barrier membrane is intended to exclude the gingival tissues and promote wound repopulation by PDL cells. The use of grafts is intended to provide a scaffold for wound repopulation by PDL cells, and in some cases it may trigger osteogenesis. The addition of biological agents such as enamel matrix proteins or rhPDGF-BB are intended to direct cellular wound repopulation and appropriate differentiation outcomes.

Although there is evidence supporting the efficacy of these techniques in promoting periodontal regeneration, their overall effects are modest [6-8]. Moreover, they are applicable only in very specific circumstances, specifically in narrow contained infrabony defects and class II furcation lesions. In addition, although these techniques address the issue of regeneration of deep periodontal tissues, they do not reverse the poor aesthetics resulting from the gingival recession and loss of interdental papillae typically seen in periodontitis. There is little evidence that any of these current techniques show consistent significant benefits compared to others, and indeed, combinations of the techniques do not generally show markedly improved efficacy, with the possible exception of the use of PDGF combined with alloplastic $\beta$-tricalcium phosphate graft [9]. Interestingly, in recent studies of treatment of highly contained narrow infrabony defects, a minimally invasive surgical approach without any additional regenerative therapies appears to provide equivalent results to those in which treatment with enamel matrix proteins or alloplastic grafting were also used [10, 11].

The current status of periodontal regenerative therapies establishes the base from which any stem cell-based therapy needs to improve. The treatment of non-contained bony defects, regeneration of supracrestal periodontal, and regeneration of gingival soft tissue appear to be particularly critical long-term goals upon which to judge potential novel regenerative therapies. 


\section{Mesenchymal Stem Cells}

Stem cells are undifferentiated cells that are defined by their properties of self-renewal and differentiation potential - the ability to give rise to a range of mature cell phenotypes. They may be isolated from embryonic tissue (embryonic stem cells) or from adult tissues (somatic stem cells). Embryonic stem (ES) cells can be maintained indefinitely and are totipotent, meaning they have the capacity to give rise to all cell phenotypes. Somatic stem cells have been isolated from a wide range of adult tissues and, in general, have a more restricted repertoire of differentiation potential (i.e., are multipotent). They exhibit the capacity for extensive self-renewal but have a finite lifespan.

In addition, a third type of stem cell, the induced pluripotent stem cell (iPS), has now been reported. iPS cells were first described following the stable transfection of embryonic and adult mouse fibroblasts with genes associated with ES cells, including Oct3/4, Sox-2, c-Myc and Klf-4 $[12,13]$. Since that time, iPS cells have been produced from many human cell types, including gingival and periodontal fibroblasts [14-16]. As they demonstrate many of the same properties as ES cells, including a very wide range of differentiation potential, iPS cells have garnered much interest within the stem cell research community for a number of reasons, not the least of which is the absence of ethical issues surrounding the generation of ES cells.

Mesenchymal stem cells are somatic stem cells that were originally described by Friedenstien and colleagues as fibroblastic cells derived from bone marrow stroma with the ability to form colonies in vitro and with the potential to differentiate to form bone, cartilage, and adipose tissue [17]. In 2006, the International Society for Cellular Therapy proposed minimum criteria to define MSCs, the first of which was that MSCs must be plastic-adherent in certain cultures. Secondly, they must express the surface markers CD105, CD73 and CD90, and must not express CD45, CD34, CD14, CD19, or HLA-DR. Thirdly, they must be able to differentiate into osteoblasts, adipocytes, and chondroblasts in vitro [18]. In addition to these criteria, MSCs exhibit self-renewal and are clonogenic. MSCs exhibiting these properties have now been isolated from a wide range of tissues, including bone marrow, adipose tissue, fetal liver and blood, Wharton's Jelly of the umbilical cord, and dental tissues, including periodontal ligament (PDL MSC), dental pulp (DPSC), exfoliated deciduous teeth (SHED cells), and dental follicle [4, 19-21]. Recent studies have also described the isolation and characterization of MSCs from healthy and inflamed gingival tissue by isolation of clonogenic cells or magnet immunosorting, and this has been proposed as a potentially more abundant source of tissue for deriving autologous MSCs when compared to PDL [22-24]. A recent study also suggested that gingiva-derived MSCs include both neural crest-derived and mesodermally derived cells [25•].
Phenotype of PDL MSCs

Although these various tissue-derived MSCs may share many properties, an important question arises as to whether they are phenotypically distinct. Within the field of periodontal regeneration, in particular, a number of studies have aimed to investigate the specific phenotypic characteristics of periodontal ligamentderived MSCs. This may be the result of observations that PDL MSCs can give rise to tissues resembling bone, cementum, and periodontal ligament when implanted in nude mouse models. Methodologically, these observations are hampered by the absence of truly specific markers of PDL and cementum. However, it is known that certain genes such as periostin, periodontal ligament-associated protein (PLAP), and scleraxis are highly expressed in the PDL, and that cementum expresses bone matrix proteins, including bone sialoprotein, and is high in cementum attachment protein and cementum protein 1 (CEMP1), which may also be found in bone [26, 27]. Given this caveat, there is a risk that some of these observations may be subject to inadvertent bias, particularly when based on histological appearance, whereby observation of mineralized tissue is described as "cementum-like" based on the finding of mineralized tissue with associated connective tissue fibers.

Nevertheless, a number of studies do suggest significant phenotypic differences between PDL MSC and MSCs derived from other sources in vitro, despite the fact that they carry the same immunological surface markers. Studies have reported increased proliferative potential and osteoblastic differentiation from PDL and other dental stem cells when compared with bone marrow stromal cells (BMSC) in vitro, and have suggested their increased potential for neurogenic differentiation, possibly reflecting their neural crest origin $[28,29,30$, 31]. In addition, preliminary studies using microarray analysis of gene expression have described marked quantitative differences in gene expression patterns between BMSC, dental pulp stem cells, and PDL MSC [32].

Two interesting recent studies investigated differences in the proteomes of MSCs of distinct sources. Using mass spectrometric analysis, Mrozik and colleagues investigated proteomic differences between BMSC, DPSC, and PDL MSC derived from the same (ovine) donor and reported 58 proteins that were differentially expressed among the different MSC sources [33]. Similarly, Eleuterio and colleagues carried out mass spectrometric analysis of proteomic differences between PDL MSC and matched DPSC and from BMSC (derived from a difference source) [34•]. This study reported a number of marked quantitative differences in protein expression among MSC populations, although the expression profiles were qualitatively quite similar.

Collectively, these studies support the hypothesis that PDL MSCs are phenotypically distinct when tested in vitro. There are a number of possible explanations for these observations. Firstly, the cells may have inherently different properties, such 
as differentiation potentials, and this may be because of their embryological (neural crest) origins [35-38]. Interestingly, however, in a study using GFP-labeled BMSCs for lineage tracing, Zhou and colleagues reported that BMSCs contribute to the total MSC population within the PDL and can differentiate into dental-specific tissues, suggesting regulation by local environmental conditions. Secondly, despite the persistence of the characteristic cell surface markers of MSCs, MSC cultures are heterogeneous, containing cells of more restricted lineage potential, and may show reduced stemness and proliferative capacities with aging both in terms of donor age and time in culture [39-42]. Consequently, studies of phenotypic differences between cells from different donors should be interpreted with caution. Studies using matched cultures derived simultaneously from the same donor may therefore be particularly valuable models in which to investigate true phenotypic differences between cells [33, 43].

With respect to the goal of utilizing MSC for therapeutic application in periodontal regeneration, phenotypic differences in vitro between MSCs of different origin may not necessarily translate to superior clinical outcomes when using PDL MSC compared to other cell types. These questions will require specific studies, including animal models of regeneration, as further considered below.

\section{Immunomodulatory Properties of PDL MSCs}

It has been known for a number of years that MSCs derived from bone marrow exert powerful immunosuppressive effects [44]. These properties have 2 potentially important clinical implications - firstly, that transplanted MSCs might be utilized as specific immunosuppressive therapeutic agents for the management of autoimmune diseases, and secondly, that implantation of allogeneic MSCs might be used in regenerative procedures rather than requiring the use of autologous cells isolated from the patient and expanded ex vivo prior to the procedure. It has now become clear that dental MSCs, including those derived from PDL, exhibit similar immunomodulatory properties to those of BMSCs. Immunomodulation by dental MSCs was first described by Wada and colleagues, who showed that these cells produced soluble factors, including TGF- $\beta 1$, hepatocyte growth factor (HGF), and indoleamine 2,3-dioxygenase (IDO), which resulted in inhibition of mitogen-induced $\mathrm{T}$ cell proliferation. Ding and colleagues demonstrated that porcine PDL MSCs failed to elicit a T cell proliferative response and did not express human leucocyte antigen (HLA)-II DR, CD80, and CD 86. They further demonstrated that PDL MSCs inhibited mitogen-stimulated T cell proliferative responses via a PGE2-mediated mechanism [45]. This study, along with a recently published study using an ovine model, have now demonstrated successful transplantation and survival in animal studies of periodontal regeneration without induction of an inflammatory response, suggesting the potential suitability of allogeneic PDL cells for use in therapeutic development of regenerative procedures [45]. The broader subject of mechanisms of immunomodulatory properties of MSCs, including those derived from dental tissues, was the subject of a recent excellent review, but this is beyond the scope of this article [46].

\section{Preclinical Models - Animal Studies}

Studies of MSCs demonstrate the potential to isolate multipotent cells which can differentiate into the tissues of the PDL both in vitro and in ectopic implantation animal models, typically using nude mice. There are, of course, a number of important challenges that need to be addressed to translate these basic findings into successful tissue engineering strategies to achieve periodontal regeneration in the clinic. These issues include optimization of cell sources, how cells are expanded ex vivo prior to implantation, choice of delivery system/scaffold for implantation, and ultimately the efficacy and magnitude of effect of the proposed treatments. These questions pose difficult practical problems, involving the use of animal models, for example, to compare different cell sources and delivery systems.

In terms of the practicality of ex vivo expansion, it is known that expansion can be greatly enhanced by appropriate medium supplements [47], suggesting the feasibility of utilizing chemically defined serum-free medium [48•]. A recent study with human BMSC has shown that use of FGF2, PDGF, or ascorbate can increase by over 1000 -fold the total number of cells generated while retaining their stem cell properties [42]. In addition, it has been demonstrated that the age of the donor may have a marked effect on the capacity for ex vivo expansion of MSCs [49].

\section{Use of MSC for Periodontal Regeneration in Animal Models}

The choice of animal model for preclinical PDL regenerative studies - albeit essentially for "proof of principle" studies may significantly influence findings. Of course, findings in animal models may be difficult at times to translate to human studies. However, a wide range of animal models of periodontal regeneration have been used, including rodent, canine, ovine, and porcine models, that have demonstrated beneficial effects on PDL and mineralized tissue formation [4, 24, 50, $51 \bullet, 52,53,54 \bullet \cdot, 55]$. The majority of these studies utilized surgically created infrabony defects. Liu and colleagues also described the use of a periodontitis model in minipigs by first surgically creating a defect in the interdental region and then placing a silk ligature to induce a periodontal lesion prior to placement of the stem cell treatment $[45,56]$.

A range of different cell sources, including PDL MSC [54••], DPSC [55], adipose tissue [52], BMSC [57], and gingival MSC, have demonstrated the ability to increase 
regeneration of periodontal tissues [24]. Although these studies have not demonstrated superior results of any particular cell type over another, PDL cells were found to have superior outcomes in a study by Park and colleagues that tested the effects of autologous PDL, DPSC, and follicular stem cells on regeneration of defects extending to the root apex in a canine model [58].

Most of these studies have employed autologous cells that have been isolated, expanded ex vivo, and reimplanted. However, given the immunosuppressive effects of MSCs as previously described, the possibility of utilizing allogeneic cells for implantation is an attractive alternative. Ding and colleagues compared the regenerative capacity of autologous and allogeneic PDL MSCs in the minipig periodontitis model and found equivalent regenerative outcomes [45]. In recent studies, allogeneic SHED cells were also shown to promote regeneration in the minipig model [59], and allogeneic PDL cells were able to enhance regeneration in a rat fenestration defect model [51•]. Mrozik and colleagues utilized an ovine model of regeneration using surgically created uncontained buccal bony defects to test the effect of allogeneic PDL cells in a Gelfoam carrier [50•]. Although the cells were well tolerated and showed trends towards more periodontal regeneration, this was not significantly greater than the use of the Gelfoam carrier alone (which demonstrated a statistically significant increase of regeneration compared to control defects) [50 ].

\section{Inducible PS Cells and ES Cells}

In addition to the MSCs, embryonic stem (ES) cells and iPS cells have also been investigated in a small number of periodontal regeneration studies. Duan and colleagues described the implantation of iPS cells combined with enamel matrix derivative (EMD) into rat periodontal fenestration defects, which demonstrated increase bone, cementum, and PDL formation with both EMD and EMD+iPS cells, with slightly greater effects seen with iPS treatment [60]. In a recent study, Hynes and colleagues reported the induction of iPS into MSC and subsequent implantation of iPS-derived MSCs into rat fenestration defects, resulting in enhancement of bone, cementum, and periodontal regeneration, with particular enhancement of the complete "bridging" of the bony fenestration defects [61•]. The induction of iPS into MSC as a precursor for use in regenerative studies may be an important step in order to avoid the potential risks of implantation of totipotent cells.

Yang and colleagues recently reported the use of ES cell transplantation in a minipig periodontitis model that demonstrated improved clinical periodontal parameters (approximately $1.5 \mathrm{~mm}$ extra pocket depth reduction and approximately $2 \mathrm{~mm}$ increased CAL gain compared to controls) and improved cementum formation, although this was not quantified histomorphometrically [53]. Furthermore, by using GFP labeling of cells prior to transplantation, they demonstrated persistence of ES cells and incorporation of cells into regenerated tissue, suggesting that the ES cells contributed directly to the tissue formation process. In addition, some transplanted cells became incorporated into distant organs and tissues, suggesting systemic cell transmission. Aside from ethical issues of ES cell use, the totipotent nature of these cells does raise important safety issues and the possibility of teratoma formation in such models, although this was not observed here.

\section{Contribution of Transplanted Cells to Tissue Regeneration}

In most of the above-described studies, the precise contribution of stem cells implanted into wounds is an important consideration, particularly as it relates to transplanted MSCs. This is especially the case given the increasing evidence that intravenous administration of MSCs can enhance clinical regenerative outcomes in a number of conditions despite the apparent lack of persistence of these cells or their incorporation into newly formed tissues [62, 63]. In these cases, it is postulated that transplanted MSCs are acting principally through mechanisms involving the paracrine regulation of immune responses and by release of trophic cytokines that direct endogenous cell regeneration. Additionally, the possibility of crosstalk between implanted MSCs and local cells has been described in vitro [64].

Clearly, there are potentially important differences between the local implantation of MSCs in a periodontal wound and the systemic administration of MSCs, and this remains an important issue in stem cell-mediated PDL regeneration. In an attempt to investigate this difference, $\mathrm{Yu}$ and colleagues recently reported their study of transplantation of GFP-labeled PDL MSC in gelatin sponges in a rat infrabony defect model using GFP-labeled gingival fibroblasts as controls. PDL MSC-treated defects showed increased bone and PDL regeneration. Rather strikingly, PDL-labeled MSCs were specifically densely located adjacent to the regenerated tissue, suggestive of cell homing to the defect, whereas gingival cells were sparsely distributed throughout the tissue. However, there were few labeled cells incorporated into the new tissue, and the authors suggest that the improved outcomes may be largely the result of paracrine regulation of endogenous cells.

\section{Delivery Systems for Implantation of Cells}

In the studies described above, a wide range of different carrier systems and scaffolds have been utilized for the delivery of stem cells. These range from the very simple cell sheets to rather complex 3D structures. Many studies have successfully utilized collagen, gelatin, or fibrin sponges [54••, 65-67]. Other studies have variously reported the use of hydroxyapatite/tricalcium phosphate [56], alginate hydrogels [68], silk scaffolds [60], amniotic membrane [69], and increasingly, the use of cell sheets utilizing the matrix produced 
in vitro to deliver cells [70-72]. In a recent development, Lee and colleagues reported the production of $3 \mathrm{D}$ multiphase scaffolds by $3 \mathrm{D}$ printing techniques capable of delivering bioactive molecules in a temporospatial-specific fashion [73•]. At this time, there is no clear understanding of the optimal properties for a cell delivery system for periodontal regeneration, particularly given the apparent successful use of these highly disparate methods. It is possible that sophisticated scaffolds such as described by Lee and colleagues may have particular application when considering challenging clinical treatments such as the need to treat suprabony defects or to regenerate periodontal and gingival soft tissue defects.

\section{Conclusions}

Over the past 5 years there has been an almost exponential increase in publications on the subject of stem cell-mediated periodontal regeneration. The evidence suggests that PDL MSC may represent a unique MSC phenotype, although it does not suggest that these cells are uniquely capable of periodontal regeneration. PDL MSCs, like other MSCs, show powerful immunomodulatory properties, and indeed, some preliminary evidence suggests that the major role of MSCs in periodontal regeneration may be by paracrine regulation rather than direct contribution to the regenerating tissues. Although the application of allogeneic MSCs and production of MSCs from iPS cells may prove to be important practical developments in the field, many issues remain to be solved in order to produce clinically useful outcomes capable of significantly extending the potential for periodontal regenerative treatment techniques in a broad range of clinical settings.

\section{Compliance with Ethics Guidelines}

Conflict of Interest Dr. Francis Hughes declares no potential conflict of interest relevant to this article.

Human and Animal Rights and Informed Consent This article does not contain any studies with human or animal subjects performed by any of the authors.

\section{References}

Papers of particular interest, published recently, have been highlighted as:

- Of importance

- Of particular importance

1. Karring T, Isidor F, Nyman S, Lindhe J. New attachment formation on teeth with a reduced but healthy periodontal ligament. J Clin Periodontol. 1985;12:51-60.
2. Melcher AH. On the repair potential of periodontal tissues. $\mathrm{J}$ Periodontol. 1976;47:256-60.

3. Gottlow J, Nyman S, Karring T, Lindhe J. New attachment formation as the result of controlled tissue regeneration. J Clin Periodontol. 1984;11:494-503.

4. Seo BM, Miura M, Gronthos S, et al. Investigation of multipotent postnatal stem cells from human periodontal ligament. Lancet. 2004;364:149-55.

5. Gay IC, Chen S, MacDougall M. Isolation and characterization of multipotent human periodontal ligament stem cells. Orthod Craniofac Res. 2007;10:149-60.

6. Bosshardt DD, Sculean A: Does periodontal tissue regeneration really work? Periodontol 2000 2009, 51:208-219.

7. Esposito M, Grusovin MG, Papanikolaou $\mathrm{N}$ et al.: Enamel matrix derivative (Emdogain $(\mathrm{R}))$ for periodontal tissue regeneration in intrabony defects. Cochrane Database Syst Rev 2009, CD003875.

8. Needleman IG, Worthington HV, Giedrys-Leeper E, Tucker RJ: Guided tissue regeneration for periodontal infra-bony defects. Cochrane Database Syst Rev 2006, CD001724.

9. Trombelli L, Farina R. Clinical outcomes with bioactive agents alone or in combination with grafting or guided tissue regeneration. J Clin Periodontol. 2008;35:117-35.

10. Trombelli L, Simonelli A, Pramstraller M, et al. Single flap approach with and without guided tissue regeneration and a hydroxyapatite biomaterial in the management of intraosseous periodontal defects. J Periodontol. 2010;81:1256-63.

11. Cortellini P, Tonetti MS. Clinical and radiographic outcomes of the modified minimally invasive surgical technique with and without regenerative materials: a randomized-controlled trial in intra-bony defects. J Clin Periodontol. 2011;38:365-73.

12. Takahashi K, Yamanaka S. Induction of pluripotent stem cells from mouse embryonic and adult fibroblast cultures by defined factors. Cell. 2006;126:663-76.

13. Takahashi K, Tanabe K, Ohnuki M, et al. Induction of pluripotent stem cells from adult human fibroblasts by defined factors. Cell. 2007;131:861-72.

14. Egusa H, Okita K, Kayashima H, et al. Gingival fibroblasts as a promising source of induced pluripotent stem cells. PLoS One. 2010;5:e12743.

15. Wada N, Wang B, Lin NH, et al. Induced pluripotent stem cell lines derived from human gingival fibroblasts and periodontal ligament fibroblasts. J Periodontal Res. 2011;46:438-47.

16. Nomura Y, Ishikawa M, Yashiro Y, et al. Human periodontal ligament fibroblasts are the optimal cell source for induced pluripotent stem cells. Histochem Cell Biol. 2012;137:719-32.

17. Friedenstein AJ, Deriglasova UF, Kulagina NN, et al. Precursors for fibroblasts in different populations of hematopoietic cells as detected by the in vitro colony assay method. Exp Hematol. 1974;2:83-92.

18. Dominici M, Le Blanc K, Mueller I, et al. Minimal criteria for defining multipotent mesenchymal stromal cells. The International Society for Cellular Therapy position statement. Cytotherapy. 2006;8:315-7.

19. Campagnoli C, Roberts IA, Kumar S, et al. Identification of mesenchymal stem/progenitor cells in human first-trimester fetal blood, liver, and bone marrow. Blood. 2001;98:2396-402.

20. Yu S, Long J, Yu J, et al. Analysis of differentiation potentials and gene expression profiles of mesenchymal stem cells derived from periodontal ligament and Wharton's jelly of the umbilical cord. Cells Tissues Organs. 2013;197:209-23.

21. Broccaioli E, Niada S, Rasperini G, et al. Mesenchymal Stem Cells from Bichat's Fat Pad: In Vitro Comparison with Adipose-Derived Stem Cells from Subcutaneous Tissue. Biores Open Access. 2013;2:107-17.

22. Ge S, Mrozik KM, Menicanin D, et al. Isolation and characterization of mesenchymal stem cell-like cells from healthy and inflamed 
gingival tissue: potential use for clinical therapy. Regen Med. 2012;7:819-32.

23. Yang H, Gao LN, An Y, et al. Comparison of mesenchymal stem cells derived from gingival tissue and periodontal ligament in different incubation conditions. Biomaterials. 2013;34:7033-47.

24. Fawzy El-Sayed KM, Paris S, Becker ST, et al. Periodontal regeneration employing gingival margin-derived stem/progenitor cells: an animal study. J Clin Periodontol. 2012;39:861-70.

25. Xu X, Chen C, Akiyama K, et al. Gingivae contain neural-crest- and mesoderm-derived mesenchymal stem cells. J Dent Res. 2013;92: 825-32. Interesting characterisation of MSC derived from gingival tissue.

26. Horiuchi K, Amizuka N, Takeshita S, et al. Identification and characterization of a novel protein, periostin, with restricted expression to periosteum and periodontal ligament and increased expression by transforming growth factor beta. J Bone Miner Res. 1999;14:1239-49.

27. Yamada S, Ozawa Y, Tomoeda M, et al. Regulation of PLAP-1 expression in periodontal ligament cells. J Dent Res. 2006;85:447-51.

28. Tamaki Y, Nakahara T, Ishikawa H, Sato S. In vitro analysis of mesenchymal stem cells derived from human teeth and bone marrow. Odontology. 2013;101:121-32.

29. Huang GT, Gronthos S, Shi S. Mesenchymal stem cells derived from dental tissues vs. those from other sources: their biology and role in regenerative medicine. J Dent Res. 2009;88:792-806.

30. Tomokiyo A, Maeda H, Fujii S, et al. A multipotent clonal human periodontal ligament cell line with neural crest cell phenotypes promotes neurocytic differentiation, migration, and survival. J Cell Physiol. 2012;227:2040-50. Demonstration of neurogenic capacity of a PDL derived MSC clone.

31. Iwata T, Yamato M, Zhang Z, et al. Validation of human periodontal ligament-derived cells as a reliable source for cytotherapeutic use. $\mathrm{J}$ Clin Periodontol. 2010;37:1088-99.

32. Kim SH, Kim YS, Lee SY, et al. Gene expression profile in mesenchymal stem cells derived from dental tissues and bone marrow. J Periodontal Implant Sci. 2011;41:192-200.

33. Mrozik KM, Zilm PS, Bagley CJ, et al. Proteomic characterization of mesenchymal stem cell-like populations derived from ovine periodontal ligament, dental pulp, and bone marrow: analysis of differentially expressed proteins. Stem Cells Dev. 2010;19:1485-99.

34. Eleuterio E, Trubiani O, Sulpizio M, et al. Proteome of human stem cells from periodontal ligament and dental pulp. PLoS One. 2013;8: e71101. Interesting comparative study of protein expression profiles of PDL and dental pulp MSCs.

35. Coura GS, Garcez RC, de Aguiar CB, et al. Human periodontal ligament: a niche of neural crest stem cells. J Periodontal Res. 2008;43:531-6.

36. Fortino VR, Chen RS, Pelaez D, Cheung HS: Neurogenesis of neural crest derived periodontal ligament stem cells by EGF and bFGF. J Cell Physiol 2014;229;479-88

37. Huang CY, Pelaez D, Dominguez-Bendala J, et al. Plasticity of stem cells derived from adult periodontal ligament. Regen Med. 2009;4:809-21.

38. Luan $\mathrm{X}$, Dangaria $\mathrm{S}$, Ito $\mathrm{Y}$, et al. Neural crest lineage segregation: a blueprint for periodontal regeneration. J Dent Res. 2009;88:781-91.

39. Singhatanadgit $\mathrm{W}$, Donos N, Olsen I. Isolation and characterization of stem cell clones from adult human ligament. Tissue Eng A. 2009;15:2625-36.

40. Lossdörfer S, Kraus D, Jäger A. Aging affects the phenotypic characteristics of human periodontal ligament cells and the cellular response to hormonal stimulation in vitro. J Periodontal Res. 2010;45:764-71.

41. Gharibi B, Ghuman MS, Hughes FJ. Akt- and Erk-mediated regulation of proliferation and differentiation during PDGFRbetainduced MSC self-renewal. J Cell Mol Med. 2012;16:2789-801.
42. Gharibi B, Hughes FJ. Effects of medium supplements on proliferation, differentiation potential, and in vitro expansion of mesenchymal stem cells. Stem Cells Transl Med. 2012;1:771-82.

43. Chen K, Xiong H, Huang Y, Liu C. Comparative analysis of in vitro periodontal characteristics of stem cells from apical papilla (SCAP) and periodontal ligament stem cells (PDLSCs). Arch Oral Biol. 2013;58:997-1006.

44. Bartholomew A, Sturgeon C, Siatskas M, et al. Mesenchymal stem cells suppress lymphocyte proliferation in vitro and prolong skin graft survival in vivo. Exp Hematol. 2002;30:42-8.

45. Ding G, Liu Y, Wang W, et al. Allogeneic periodontal ligament stem cell therapy for periodontitis in swine. Stem Cells. 2010;28: 1829-38.

46. Wada N, Gronthos S, Bartold PM: Immunomodulatory effects of stem cells. Periodontol 2000 2013, 63:198-216.

47. Jung IH, Kwon BS, Kim SH, et al. Optimal medium formulation for the long-term expansion and maintenance of human periodontal ligament stem cells. J Periodontol. 2013;84:1434-44.

48. Tarle SA, Shi S, Kaigler D. Development of a serum-free system to expand dental-derived stem cells: PDLSCs and SHEDs. J Cell Physiol. 2011;226:66-73. Addresses an important issue in translational MSC research - that of utilising chemically defined media for in-vitro expansion of cells.

49. Zhang J, An Y, Gao LN, et al. The effect of aging on the pluripotential capacity and regenerative potential of human periodontal ligament stem cells. Biomaterials. 2012;33:6974-86.

50. Mrozik KM, Wada N, Marino V, et al. Regeneration of periodontal tissues using allogeneic periodontal ligament stem cells in an ovine model. Regen Med. 2013;8:711-23.

51. Han J, Menicanin D, Marino V et al.: Assessment of the regenerative potential of allogeneic periodontal ligament stem cells in a rodent periodontal defect model. J Periodontal Res 2013. Collectively these 2 papers establish the principle of using allogeneic cells for PDL transplantation. doi:10.1111/jre.12111.

52. Tobita M, Uysal AC, Ogawa R, et al. Periodontal tissue regeneration with adipose-derived stem cells. Tissue Eng A. 2008;14:945-53.

53. Yang JR, Hsu CW, Liao SC, et al. Transplantation of embryonic stem cells improves the regeneration of periodontal furcation defects in a porcine model. J Clin Periodontol. 2013;40:364-71.

54.• Yu N, Oortgiesen DA, Bronckers AL, et al. Enhanced periodontal tissue regeneration by periodontal cell implantation. J Clin Periodontol. 2013;40:698-706. Very interesting results obtained from using GFP-labeled cells to establish cell fate. Demonstrates evidence of homing to periodontal would and also suggests that the transplanted cells may actually contribute rather little directly to the regenerated tissue.

55. Khorsand A, Eslaminejad MB, Arabsolghar M, et al. Autologous dental pulp stem cells in regeneration of defect created in canine periodontal tissue. J Oral Implantol. 2013;39:433-43.

56. Liu Y, Zheng Y, Ding G, et al. Periodontal ligament stem cellmediated treatment for periodontitis in miniature swine. Stem Cells. 2008;26:1065-73.

57. Kim SH, Kim KH, Seo BM, et al. Alveolar bone regeneration by transplantation of periodontal ligament stem cells and bone marrow stem cells in a canine peri-implant defect model: a pilot study. J Periodontol. 2009;80:1815-23.

58. Park JY, Jeon SH, Choung PH. Efficacy of periodontal stem cell transplantation in the treatment of advanced periodontitis. Cell Transplant. 2011;20:271-85.

59. Fu X, Jin L, Ma P et al.: Allogeneic Stem Cells From Deciduous Teeth Mediated Treatment for Periodontitis in Miniature Swine. J Periodontol 2013. doi:10.1902/jop.2013.130254.

60. Duan X, Tu Q, Zhang J, et al. Application of induced pluripotent stem (iPS) cells in periodontal tissue regeneration. J Cell Physiol. 2011;226:150-7. 
61. Hynes K, Menicanin D, Han J, et al. Mesenchymal stem cells from iPS cells facilitate periodontal regeneration. J Dent Res. 2013;92: 833-9. Demonstrates the generation of MSC cells from iPS cells and their potential role in periodontal regeneration.

62. Shin L, Peterson DA. Human mesenchymal stem cell grafts enhance normal and impaired wound healing by recruiting existing endogenous tissue stem/progenitor cells. Stem Cells Transl Med. 2013;2:33-42.

63. Quertainmont R, Cantinieaux D, Botman O, et al. Mesenchymal stem cell graft improves recovery after spinal cord injury in adult rats through neurotrophic and pro-angiogenic actions. PLoS One. 2012; 7:e39500.

64. Proksch S, Steinberg T, Stampf S, et al. Crosstalk on cell behavior in interactive cocultures of hMSCs with various oral cell types. Tissue Eng A. 2012;18:2601-10.

65. Suaid FF, Ribeiro FV, Gomes TR, et al. Autologous periodontal ligament cells in the treatment of Class III furcation defects: a study in dogs. J Clin Periodontol. 2012;39:377-84.

66. Trubiani O, Orsini G, Zini N, et al. Regenerative potential of human periodontal ligament derived stem cells on three-dimensional biomaterials: a morphological report. J Biomed Mater Res A. 2008;87: 986-93.

67. Grimm WD, Dannan A, Becher S, et al. The ability of human periodontium-derived stem cells to regenerate periodontal tissues: a preliminary in vivo investigation. Int J Periodontics Restor Dent. 2011;31:e94-101.

68. Moshaverinia A, Chen C, Akiyama K, et al. Alginate hydrogel as a promising scaffold for dental-derived stem cells: an in vitro study. $\mathrm{J}$ Mater Sci Mater Med. 2012;23:3041-51.

69. Iwasaki K, Komaki M, Yokoyama $\mathrm{N}$ et al.: Periodontal regeneration using periodontal ligament stem cell-transferred amnion. Tissue Eng Part A 2013. doi:10.1089/ten.tea.2013.0017.

70. Washio K, Iwata T, Mizutani M, et al. Assessment of cell sheets derived from human periodontal ligament cells: a pre-clinical study. Cell Tissue Res. 2010;341:397-404.

71. Iwata $\mathrm{T}$, Washio $\mathrm{K}$, Yoshida $\mathrm{T}$ et al.: Cell sheet engineering and its application for periodontal regeneration. J Tissue Eng Regen Med 2013. doi:10.1002/term. 1785.

72. Yang Z, Jin F, Zhang X, et al. Tissue engineering of cementum/ periodontal-ligament complex using a novel three-dimensional pellet cultivation system for human periodontal ligament stem cells. Tissue Eng C Methods. 2009;15:571-81.

73. Lee CH, Hajibendeh J, Takahiro S et al.: 3D Printed Multiphase Scaffolds for Regeneration of Periodontium Complex. Tissue Eng Part A 2013. Development of a sophisticated scaffold for periodontal regeneration that may be capable of directing regeneration events temporospatially. doi:10.1089/ten.TEA.2013.0386. 